\title{
STIELTJES DIFFERENTIAL-BOUNDARY OPERATORS
}

\author{
ALLAN M. KRALL
}

Abstract. The differential-boundary system $S$ :

$$
\begin{gathered}
L y=\left(y+H(t)[C y(0)+D y(1)]+H_{1}(t) \psi\right)^{\prime}+P(t) y . \\
A y(0)+B y(1)+\int_{0}^{1} d K(t) y(t)=0, \quad \int_{0}^{1} d K_{1}(t) y(t)=0,
\end{gathered}
$$

is discussed when set in the space $\mathscr{L}_{n}^{p}[0,1]$. The density of the domain of $L$ is discussed, and the adjoint or dual operator is derived. A discussion of selfadjoint systems follows. Necessary and sufficient conditions for $T=(1 / i) L$ to be selfadjoint in $\mathscr{L}_{n}^{2}[0,1]$ are given.

1. Introduction. Differential-boundary operators with discontinuities at a finite number of points were thoroughly discussed in [6]. Stieltjes differential-boundary operators were recently encountered by Brown and Krall [1] as dual operators when extending the work of Bryan [2]. In addition Bryan [3] had earlier derived Green's formula as well as other results for Stieltjes differential-boundary operators in the case where $H_{1}=0$.

In addition we should mention the work of Vejvoda and Tvrdy [7] which presented a lengthy discussion of the algebraic-measure theoretic properties required of $S$, and finally, the work by Halanay and Moro [4], which discussed the existence and uniqueness of solutions for $S$.

Our point of view differs somewhat from those just mentioned written by others in that we will consider $S$ as generated by a differential-boundary operator in $\mathscr{L}_{n}^{p}[0,1], 1 \leqq p<\infty$, then derive the dual operator in $\mathscr{L}_{n}^{a}[0,1], 1 / p+1 / q=1$.

Specifically, in $\mathscr{L}_{n}^{p}[0,1]$ we wish to define the operator $L$ in the following way: (1) Let $A$ and $B$ be $m \times n$ matrix constants with $m \leqq 2 n$ such that $\operatorname{rank}(A B)=m$. Let $C$ and $D$ be $(2 n-m) \times n$ matrices such that $\left(\begin{array}{l}A \\ C\end{array}\right.$ nonsingular.

(2) Let $K$ be an $m \times n$ matrix valued function of bounded variation such that, when considered as generating a matrix valued measure, $d K(0)=A, d K(1)=B$.

Received by the editors January 22, 1973.

AMS (MOS) subject classifications (1970). Primary 34B05, 34B10, 34B25; Secondary 44A60.

Key words and phrases. Operator, differential operator, adjoint operator.

(c) American Mathematical Society 1973 
(3) Let $K_{1}$ be an $r \times n$ matrix valued function of bounded variation such that, when considered as generating a matrix valued measure, $d K_{1}(0)=0, d K_{1}(1)=0$.

Thus the matrix $\left(\begin{array}{c}k \\ K_{1}\end{array}\right)$ generates an $(m+r) \times n$ matrix valued measure such that the rank of

$$
\left(\begin{array}{ll}
d K(0) & d K(1) \\
d K_{1}(0) & d K_{1}(1)
\end{array}\right)
$$

is $m$, the portion generating this rank lying in the first $m$ rows. Any arbitrary boundary condition of the form $\int_{0}^{1} d v(t) y(t)=0$ can be expressed in such a manner.

(4) Let $H$ and $H_{1}$ be $n \times(2 n-m)$ and $n \times s$ matrix valued functions of bounded variation.

(5) Let $P$ be a continuous $n \times n$ matrix.

1.1. Definition. We denote by $D$ those elements $y$ in $\mathscr{L}_{n}^{p}[0,1]$ satisfying

(a) for each $y$ there exists an $s \times 1$ matrix valued constant $\psi$ such that $y+H[C y(0)+D y(1)]+H_{1} \psi$ is absolutely continuous.

(b) $l y=\left(y+H[C y(0)+D y(1)]+H_{1} \psi\right)^{\prime}+P y$ exists a.e. and is in $\mathscr{L}_{n}^{p}[0,1]$.

(c) $A y(0)+B y(1)+\int_{0}^{1} d K(t) y(t)=0, \int_{0}^{1} d K_{1}(t) y(t)=0$.

We define the differential-boundary operator $L$ by letting $L y=l y$ for all $y$ in $D$.

Before we can proceed further we must know whether or not $D$ is dense in $\mathscr{L}_{n}^{p}[0,1]$. The adjoint or dual operator will not exist in the ordinary sense unless it is. To decide this question we introduce the notion of the kernel of a measure.

1.2 Definition. Let the measure $v$ be generated by the matrix of bounded variation $\left(\begin{array}{l}K \\ K_{1}\end{array}\right)$ so that

$$
\int_{0}^{1} d v(t) y(t)=\left(\begin{array}{c}
A y(0)+B y(1)+\int_{0}^{1} d K(t) y(t) \\
\int_{0}^{1} d K_{1}(t) y(t)
\end{array}\right) .
$$

Let $T$ be a set which is everywhere dense in $[0,1]$. For fixed the kernel of $v^{*}(t)$ is the set of vectors $v$ satisfying $\nu^{*}(t) v=0 .{ }^{1}$ By the kernel $K_{v^{*}}^{T}$ of $v^{*}$ with respect to $T$ we mean $\bigcap_{t \in T} \operatorname{ker} \nu^{*}[0, t]$.

It can be shown that if $1 \in T$, then $K_{v^{*}}^{T}$ is independent of the particular choice of $T$ when compared with kernels for other dense sets [1].

1 The ${ }^{*}$ indicates the matrix conjugate transpose. 
1.2. THEOREM. Let $\nu^{*}=v_{s}^{*}+v_{c}^{*}$, where $v_{s}^{*} \perp v_{c}^{*}, v_{s}^{*}$ is singular with respect to Lebesgue measure, and $v_{c}^{*}$ is absolutely continuous with respect to Lebesgue measure. $D$ is dense in $\mathscr{L}_{n}^{p}[0,1]$ if and only if $K_{v_{s}{ }^{*}}^{T} \subset K_{v_{c}{ }^{*}}^{T}$.

The proof is found in [1]. In our case we need only apply it to those elements in $D$ for which $\psi=0$.

We shall assume throughout the remainder of this paper that the boundary conditions are acceptable: that $D$ is dense in $\mathscr{L}_{n}^{p}[0,1]$. This implies that the dual operator does exist in $\mathscr{L}_{n}^{a}[0,1], 1 / p+1 / q=1$.

2. The dual operator. Let us denote the inverse of the matrix $\left(\begin{array}{ll}A & P \\ C & D\end{array}\right)$ by

$$
\left(\begin{array}{cc}
-\tilde{A}^{*} & -\tilde{C}^{*} \\
\tilde{B}^{*} & \tilde{D}^{*}
\end{array}\right)
$$

I.e.

$$
\left(\begin{array}{ll}
A & B \\
C & D
\end{array}\right)\left(\begin{array}{cc}
-\tilde{A}^{*} & -\tilde{C}^{*} \\
\tilde{B}^{*} & \tilde{D}^{*}
\end{array}\right)=\left(\begin{array}{cc}
-\tilde{A}^{*} & -\tilde{C}^{*} \\
\tilde{B}^{*} & \tilde{D}^{*}
\end{array}\right)\left(\begin{array}{ll}
A & B \\
C & D
\end{array}\right)=I_{2 n} .
$$

Componentwise these products imply

$$
\begin{aligned}
-A \tilde{A}^{*}+B \widetilde{B}^{*} & =I, & -A \tilde{C}^{*}+B \tilde{D}^{*} & =0, \\
-C \tilde{A}^{*}+D \widetilde{B}^{*} & =0, & -C \widetilde{C}^{*}+D \tilde{D}^{*} & =I,
\end{aligned}
$$

and

$$
\begin{array}{ll}
\tilde{A}^{*} A+\tilde{C}^{*} C=-I, & \tilde{A}^{*} B+\tilde{C}^{*} D=0, \\
\tilde{B}^{*} A+\tilde{D}^{*} C=0, & \tilde{B}^{*} B+\tilde{D}^{*} D=I,
\end{array}
$$

it being understood that $\tilde{A}, \widetilde{B}, \widetilde{C}$ and $\tilde{D}$ are $m \times n, m \times n,(2 n-m) \times n$ and $(2 n-m) \times n$ matrices.

2.1. Definition. We denote by $D^{+}$those elements $z$ in $\mathscr{L}_{n}^{a}[0,1]$ satisfying

(a) for each $z$ there exists an $r \times 1$ matrix valued constant $\phi$ such that $z+K^{*}[\tilde{A} z(0)+\widetilde{B} z(1)]+K_{1}^{*} \phi$ is absolutely continuous.

(b) $l^{+} z=-\left(z+K^{*}[\tilde{A} z(0)+\widetilde{B} z(1)]+K_{1}^{*} \phi\right)^{\prime}+P^{*} z$ exists a.e. and is in $\mathscr{L}_{n}^{a}[0,1]$.

(c) $\widetilde{C}_{z}(0)+\tilde{D} z(1)+\int_{0}^{1} d H^{*}(t) z(t)=0, \int_{0}^{1} d H_{1}^{*}(t) z(t)=0$.

We define the differential-boundary operator $L^{+}$by letting $L^{+} z=l^{+} z$ for all $z$ in $D^{+}$.

Our aim is to show that the dual of the operator $L$ is $L^{+}$. In order to do so, however, it is necessary to have two forms of Green's formula available to us. 
Let $D_{0}$ denote those elements $y$ in $\mathscr{L}_{n}^{p}[0,1]$ satisfying:

(a) For each $y$ there exists an $s \times 1$ matrix valued constant $\psi$ such that $y+H[C y(0)+D y(1)]+H_{1} \psi$ is absolutely continuous.

(b) $l y=\left(y+H[C y(0)+D y(1)]+H_{1} \psi\right)^{\prime}+P y$ exists a.e. and is in $\mathscr{L}_{n}^{p}[0,1]$.

Let $D_{0}^{+}$denote those elements $z$ in $\mathscr{L}_{n}^{a}[0,1]$ satisfying:

(a) For each $z$ there exists an $r \times 1$ matrix valued constant $\phi$ such that $z+K^{*}[\tilde{A} z(0)+\widetilde{B} z(1)]+K_{1}^{*} \phi$ is absolutely continuous.

(b) $l^{+} z=-\left(z+K^{*}[\tilde{A} z(0)+\tilde{B} z(1)]+K_{1}^{*} \phi\right)^{\prime}+P^{*} z$ exists a.e. and is in $\mathscr{L}_{n}^{a}[0,1]$.

Let $D_{0}^{++}$denote those elements $z$ in $\mathscr{L}_{n}^{q}[0,1]$ satisfying:

(a) For each $z$ there exist an $r \times 1$ matrix valued constant $\phi$ and an $m \times 1$ matrix valued constant $\phi_{1}$ such that $z+K^{*} \phi_{1}+K_{1}^{*} \phi$ is absolutely continuous.

(b) $l^{++} z=-\left(z+K^{*} \phi_{1}+K_{1}^{*} \phi\right)^{\prime}+P^{*} z$ exists a.e. and is in $\mathscr{L}_{n}^{a}[0,1]$.

The following two results can be easily derived (see Bryan [3], Vejvoda and Tvrdy [7]).

2.2. Theorem (Green's formula). Let $y$ be in $D_{0}, z$ be in $D_{0}^{+}$. Then

$$
\begin{aligned}
\int_{0}^{1}\left[z^{*}(l y)-\left(l^{+} z\right)^{*} y\right] d t \\
=\left[\tilde{C} z(0)+\tilde{D} z(1)+\int_{0}^{1} d H^{*} z\right]^{*}[C y(0)+D y(1)] \\
+[\tilde{A} z(0)+\tilde{B} z(1)]^{*}\left[A y(0)+B y(1)+\int_{0}^{1} d K y\right] \\
\quad+\phi^{*}\left[\int_{0}^{1} d K_{1} y\right]+\left[\int_{0}^{1} d H_{1}^{*} z\right]^{*} \psi .
\end{aligned}
$$

2.3. TheOREm. Let $y$ be in $D_{0}, z$ be in $D_{0}^{++}$. Then

$$
\begin{aligned}
\int_{0}^{1}\left[z^{*}(l y)=\right. & \left.\left(l^{++} z\right)^{*} y\right] d t \\
= & {\left[\tilde{C} z(0)+\tilde{D} z(1)+\int_{0}^{1} d H^{*} z\right]^{*}[C y(0)+D y(1)] } \\
& +[\tilde{A} z(0)+\tilde{B} z(1)]^{*}\left[A y(0)+B y(1)+\int_{0}^{1} d K y\right] \\
& +\phi^{*}\left[\int_{0}^{1} d K_{1} y\right]+\left[\int_{0}^{1} d H_{1}^{*} z\right]^{*} \psi \\
& +\left[\phi_{1}-(\tilde{A} z(0)+\widetilde{B} z(1))\right]^{*}\left[\int_{0}^{1} d K y\right] .
\end{aligned}
$$

Remarks are now in order concerning the form of these operators. 
We shall say that an operator of the form $(y+H[C y(0)+D y(1)])^{\prime}+P y$ is endpoint restricted because of the nature of the adjusting term $H[C y(0)+D y(1)]$. An operator of the form $\left(y+H_{1} \psi\right)^{\prime}+P y$ is free since $\psi$ is undetermined by specific points. Operators of the form given by $l$ are mixed in form. There exists a multitude of other mixed operators: For instance, the operators discussed in [6] are of the form

$$
\left(y+\sum_{i=1}^{m} H_{i}\left[\sum_{j=1}^{m}\left(C_{i j} y\left(a_{j-1}+\right)+D_{i j} y\left(a_{j}-\right)\right]\right)^{\prime}+P y\right.
$$

where $H_{i}, i=1, \cdots, n$, are absolutely continuous except at the discrete set of points $\left\{a_{j}\right\}_{j=1}^{m}$ are mixed.

\subsection{THEOREM. In dual of $L$ in $\mathscr{L}_{n}^{p}[0,1]$ is given by $L^{+}$in $\mathscr{L}_{n}^{q}[0,1]$.}

Proof. Let $L_{0}$ be the restriction of $L$ to those elements in $D$ which vanish at 0 and 1 and for which $\psi=0$. This operator was discussed in [1] where its dual was shown to have the form

$$
L_{0}^{*} z=l^{++} z=-\left(z+K^{*} \phi_{1}+K_{1}^{*} \phi\right)^{\prime}+P^{*} z .
$$

Since $L_{0} \subset L, L^{*} \subset L_{0}^{*}$, and $L^{*} z=-\left(z+K^{*} \phi_{1}+K_{1}^{*} \phi\right)^{\prime}+P^{*} z$. An application of Theorem 2.3 for arbitrary $y$ in $D$ shows that

and

$$
\tilde{C} z(0)+\tilde{D} z(1)+\int_{0}^{1} d H^{*} z=0, \quad \int_{0}^{1} d H_{1}^{*} z=0,
$$

$$
\phi_{1}=\tilde{A} z(0)+\widetilde{B} z(1),
$$

since the coefficients of these terms are unrestricted. This shows that $L^{*} \subset L^{+}$. The reverse inclusion is a trivial consequence of Green's formula.

2.4. Corollary. Let $y$ be in $D, z$ be in $D^{+}$, then

$$
\int_{0}^{1}\left[z^{*}(L y)-\left(L^{+} z\right)^{*} y\right] d t=0 .
$$

3. Selfadjoint systems. Let us restrict our considerations to $\mathscr{L}_{n}^{2}[0,1]$. In order to find selfadjoint differential-boundary operators it is convenient to replace the operator $L$ by

$$
T y=(1 / i)\left(y+H[C y(0)+D y(1)]+H_{1} \psi\right)^{\prime}+Q y
$$

where $(1 / i) P=Q . T^{*}$ is then given by

$$
T^{*} z=(1 / i)\left(z+K^{*}[\tilde{A} z(0)+\widetilde{B} z(1)]+K_{1}^{*} \phi\right)^{\prime}+Q^{*} z .
$$


Green's formula becomes

$$
\begin{aligned}
\int_{0}^{1}\left[z^{*}(T y)=\right. & \left.\left(T^{*} z\right)^{*} y\right] d t \\
= & (1 / i)\left\{\left[\tilde{C} z(0)+\tilde{D} z(1)+\int_{0}^{1} d H^{*} z\right]^{*}[C y(0)+D y(1)]\right. \\
& +[\tilde{A} z(0)+\tilde{B} z(1)]^{*}\left[A y(0)+B y(1)+\int_{0}^{1} d K y\right] \\
& \left.+\phi^{*}\left[\int_{0}^{1} d K_{1} y\right]+\left[\int_{0}^{1} d H_{1}^{*} z\right]^{*} \psi\right\} .
\end{aligned}
$$

We then conclude immediately:

3.1. TheOREM. T is selfadjoint in $\mathscr{L}_{n}^{2}[0,1]$ if and only if

(1) $Q=Q^{*}$.

(2) $m=n, r=s$.

(3) $K=\left[B D^{*}-A C^{*}\right] H^{*}$ a.e.

(4) $A A^{*}=B B^{*}$.

(5) $H\left[C C^{*}-D D^{*}\right]=0$ a.e.

(6) $K_{1}=M H_{1}^{*}$, where $M$ is a nonsingular $r \times r$ matrix.

The proof is quite similar to that found in [6]. Let $T$ be selfadjoint. Then $y$ absolutely continuous and vanishing at 0 and 1 (so $\psi=0, \phi=0$ also) shows $Q=Q^{*}$. A comparison of the number of boundary conditions and relative sizes of $H_{1}$ and $K_{1}$ shows $m=n, r=s$. If the elements $y$ vanish at 0 and 1 but are not necessarily absolutely continuous, then $H_{1} \psi=K_{1}^{*} \phi$, which in turn implies $K_{1}=M H_{1}^{*}$.

At this point we see that

$$
H[C y(0)+D y(1)]=K_{1}^{*}[\tilde{A} y(0)+\tilde{B} y(1)] .
$$

Since $y$ may or may not vanish at 0 or 1 , we must have $H C=K^{*} \tilde{A}$, $H D=K^{*} \widetilde{B}$. Multiplying by $C^{*}$ and $D^{*}$ and subtracting, we find $H\left[C C^{*}-D D^{*}\right]=0$. Multiplying by $A^{*}$ and $B^{*}$ and subtracting, we find $K=\left[B D^{*}-A C^{*}\right] H^{*}$.

Finally by comparing the first boundary conditions, we find $A=E \widetilde{C}$, $B=E \tilde{D}, K=E \tilde{H}^{*}$. The last we have already shown to be true. Multiplying the first by $A^{*}$, the second by $B^{*}$ and subtracting, we find $A A^{*}=B B^{*}$.

The converse is similar to that found in [6].

4. Remarks. If the spectrum of the operator $T$ is discrete, then an eigenfunction expansion like that found in [6] follows immediately. The solution of the problem $T y=\lambda y$ has not been thoroughly enough explored to say that this is definitely the case, however. The Hildebrandt 
matrix style differentio-Stieltjes-integral equation [5] which is needed seems to possess a fundamental matrix, but its exact nature as well as its analyticity in $\lambda$ has not been suitably verified. We plan to follow this paper with such a verification.

The derivation of suitable nonselfadjoint eigenfunction expansions also leads to a differentio-Stieltjes-integral equation, which in the context of the present paper involves a 5 dimensional matrix system. The same, as well as other, problems are encountered. Again we hope to present a solution in the near future.

\section{REFERENCES}

1. R. C. Brown and A. M. Krall, Ordinary differential operators under Stieltjes boundary conditions (to appear).

2. R. N. Bryan, A linear differential system with general boundary conditions, J. Differential Equations 5 (1969), 38-48. MR 38 \#1312.

3. —_ A nonhomogeneous lisear differential system with interface conditions, Proc. Amer. Math. Soc. 22 (1969), 270-276. MR 39 \#3078.

4. A. Halanay and A. Moro, $A$ boundary value problem and its adjoint, Ann. Mat. Pura Appl. (4) 79 (1968), 399-411. MR 38 \#2371.

5. T. H. Hildubrandt, On systems of linear differentio-Stieltjes-integral equations, Illinois J. Math. 3 (1959), 352-373. MR 21 \#4339.

6. A. M. Krall, Differential-boundary operators, Trans. Amer. Math. Soc. 154 (1971), 429-458. MR 42 \#6328.

7. O. Vejvoda and M. Tvrdy, Existence of solutions to a linear integro-boundarydifferential equation with additional conditions, Ann. Mat. Pura Appl. 89 (1971), 169-216.

Department of Mathematics, Pennsylvania State University, University Park, Pennsylvania 16802 